\title{
Quorum Sensing and Its Applications in Diverse Fields of Significance
}

\author{
Malik Asif Aziz ${ }^{1 *}$, Shazia Lone ${ }^{2}$, Mushtaq Ah. Malik ${ }^{1}$, Zaffar Mahdi Dar ${ }^{1}$, \\ Amjad Masood $^{3}$, Misbah Aijaz ${ }^{1}$, Saima Shafi ${ }^{1}$ and Showkat Sidique ${ }^{4}$
}

${ }^{1}$ Division of Basic Sciences \& Humanities, Faculty of Agriculture, Wadura (J\&K)-India

${ }^{2}$ Department of Environmental Sci. Govt Degree College, Kupwara ( \& K) -India

${ }^{3}$ Division of Agronomy, Faculty of Agriculture, Wadura (J\&K)-India

${ }^{4}$ Division of Statistic, Faculty of Agriculture, Wadura (J\&K)-India

*Corresponding author

\section{A B S T R A C T}

\section{Keywords}

Quorum sensing and applications

Article Info

Accepted:

12 June 2019

Available Online:

10 July 2019
Several unicellular microorganisms use small signalling molecules to find out their local concentration. The processes involved in the production and recognition of these signals are generally known as quorum sensing (QS). Unicellular microorganisms to manage their activities use this kind of cellto-cell communication, which allows them to work as multi-cellular systems. Newly, several groups have confirmed artificial intra species and inter-species communication through synthetic circuits, which incorporate components of bacterial QS systems.

\section{Introduction}

Engineered QS-based circuits have a broad range of applications such as production of biochemical's, tissue engineering, also mixedspecies fermentations. They are also extremely useful in designing microbial biosensors to identify bacterial species present in the environment and inside living organisms.

A decade ago, the secretion and perception of minor signalling molecules that in turn are transduced to coordinate behaviour of a 'smallest unit' of microorganisms was named quorum sensing by EP Greenberg with colleagues.

Ever since then, an exponential growth in understanding and occurrence of quorumsensing systems has developed, with sightings ranging from virulence inhuman along with plant pathogens to degradative capacity of activated sludge.

Advance characterization of signalling circuits, coupled with creative position applications, propose a wealth of prospects 
for advancing commercial biotechnology (March and Bentley, 2004).

Researchers in biotechnology continuously seek new platforms from which to address problems: manifesto that, in a broad sense, improve efficacy, while maintaining or intensifying specificity. Most freshly, microbial quorum sensing has emerged as such a technology.

Because microbial communities absorb a small space, concentrations of extracellular signalling molecules build up, providing motivation for unique and various cellular responses along with protection from rival microbial communities. Referred to as 'quorum sensing' for its regularly reported and concurrent dependence on high population density (Joyce et al., 2004), extracellular signalling gives a novel basis for control over molecular also cellular processes along with population behaviour, possibly in a manner more reliable with that of native physiology. Quorum sensing might be the base upon which the more complicated intracellular communication found in advanced level organisms has evolved.

Quorum sensing offers potential to create engineered bacteria capable of invading cancer cells. It is possible to envision the creation of novel anti-cancer therapeutics by the addition of cancer-destructing modules to these microbial biosensors. Another application of QS and quorum quenching lies in the creation of transgenic plants that are able to defend themselves against common bacterial pathogens. It plays a main role in controlling a diversity of microbial cell activities, such as biofilm formation and virulence that considerably impact human health, agriculture, and commercial production and transport systems. Quorum sensing is cell-to-cell communication in bacteria have ability to control development, sporulation, and antibiotic synthesis also virulence factor induction, cell differentiation, moreover nutrient flux along with extra physiological events in pathogenic bacterial infections. Scientists now a days creating more possible benefits from quorum sensing and off course there is lots of potential development for advancement in 2011ranging from marine to human disorders. Quorum Sensing considered a signalling molecule, a compound has to result a reaction in a population of cells that is different from the approach in which the cells would perform independently. There are two types of quorum sensing: species- specific and interspecies.

Species-specific quorum sensing in Gramnegative bacteria is intercede by acylhomoserine lactones (AHLs) with numerous moieties distinguishing signals between species (Fuqua et al., 2001). In Grampositive bacteria, species-specific quorum sensing is generally assist through small peptides Gram-negative bacteria like V.fischeri and Pseudomonas aeruginosa use acyl homoserine lactones (AHLs) as signals.

Components of bacterial QS systems form an important part of many artificial genetic circuits that control phenomena such as suitable behaviour, pulse response, spatialtemporal control of gene expression, and population control (Purnick and Weiss, 2009).

\section{Engineered quorum sensing systems}

The promising field of synthetic biology seeks to generate novel biological systems by applying the fundamental engineering principles of standardization and hierarchical abstraction to GE engineering (Purnick and Weiss, 2009). This method allows designers to build and optimize compound genetic circuits that perform new functions, such as DNA-damage-induced biofilm formation and preservation of synthetic ecosystems 
(Balagadde et al., 2008, Kobayashi et al., 2004). Various genetic modules can be included into complex gene networks also called "genetic systems" or "devices" using a "plug-and-play" strategy (Kobayashi et al., 2004). These gene networks are then commenced into a well-characterized, steady host cell known as a "chassis", which supplies the essential raw materials and support machinery. Operation of the artificial genetic device imparts new functionalities to the host and makes a microbial cell factory that is capable of performing preferred tasks.

Auto inducers are very useful as input signals as they are little, diffuse freely in aqueous media, and are simply taken up through cells.

As the engineered cells synthesize QS signals by themselves, they are able to watch their own cell density with modulate their activities appropriately, thereby falling the need for outer protection (Brenner et al., 2007).

Scientists have devised QS-inducible mammalian genetic circuits by mixing bacterial QS receptors with either a eukaryotic transactivation domain or with a eukaryotic transcription repressor domain (Neddermann et al., 2003; Weber and Fussenegger, 2009; Weber et al., 2005; Weber et al., 2003; Williams et al., 2004). SThese synthetic gene regulation systems will have functions in drug discovery, tissue engineering, and also industrial production of biochemical's during mammalian cell culture.

Consumption of a suitable switch module gives a pointed (ON or OFF) or binary profile of aim gene expression depending on the store concentration. Engineered QS systems including suitable switches are probable to be extremely useful in industrial production of toxic gene products and in designing environmental biosensors. Scientists have used components of the V. fischeri Quorum
Sensing system to engineer spatio-temporally keeping up cell to cell communication in $E$. coli (Basu et al., 2004). Depending on the comparative distribution of Sender and Receiver cells in a 2-D matrix, different reporter formats such as bulls' eye, ellipse, oval, heart, and clover were formed.

The capacity to develop QS type communication systems using non-Quorum Sensing signals considerably grows the design possibilities for genetic engineering systems. Through inserting the producing signal components in one species and the receptor in another, scientists have engineered inter and intra-kingdom communications among bacteria, yeast, plants, and mammalian cells (Balagadde et al., 2008, Brenner et al., 2007, Weber et al., 2007). Depending on the planned synthetic communication device, relationships like predator-prey, commensalism, mutualism, amensalism and parasitism were produced among the communicating species.

Different from engineering inter-species communication, Quorum Sensing based genetic devices can control diverse features of mixed-species fermentations. For case, based on QS population control circuits can be used to manage the cell densities of the contributing species (You et al., 2004). Based on QS gene-expression circuits can also be used to initiate expression of mark genes when the cell densities of contributing species reach a definite threshold (Brenner et al., 2007).

\section{Various applications of quorum sensing}

\section{Biosensors}

An interesting application of Quorum Sensing is in the engineering of whole cell microbial biosensors to distinguish pathogenic microbes present in the environment with diseased host 
organisms. Quorum Sensing have also been used to produce engineered bacteria capable of attacking cancer cells. It is probable to visualize the creation of new anti-cancer therapeutics by the addition of cancerdestructing elements to these microbial biosensors. Another function of QS and quorum quenching lies in the designing of transgenic plants that are able to protect themselves against general bacterial pathogens.

\section{Pathogen diagnostics and therapeutics}

The majority of the whole cell QS biosensors that have been explained so far recognize Gram-negative AHLs (Kumari et al., 2008; Steindler and Venturi, 2007). A standard AHL biosensor contains an AHL responsive transcriptional regulator also a cognate promoter, which directs the transcription of a reporter gene. It has been recommended that QS signals only can be used as markers for the occurrence of pathogenic bacteria in clinical and environmental samples. Thus, QS signals should not be engaged as the only inputs for microbial biosensors. However, Quorum sensing based amplification circuits can still be used to engineer biosensing circuits to find the occurrence of pathogenic microbes in contaminated groundwater products, dairy, and meat products.

\section{Biocontrol}

The rhizosphere is a limited region of soil that surroundings a plant's roots and is affected by secretions from the root also soil microbes in the vicinity. Quorum sensing bacteria form a main component of the rhizosphere community.

Scientists have also engaged quorumquenching enzymes to decrease bacterial virulence against plants. Though, QS systems also control necessary functions in useful rhizosphere bacteria, as well as biofilm formation, antibiotic production, and nitrogen fixation (Muller et al., 2009; SanchezContreras et al., 2007).

\section{Prevention of biofouling}

Biofouling is the increase of bacteria, algae and also animals like protozoans and crustaceans on surfaces that prolonged contact with water. Biofouling can happen on surfaces as assorted as pipes, tanks, ship hull, membrane bioreactors, medical or dental implants, and catheters.

This unwanted growth of living organisms and their secretions lead to contamination, colonization, also corrosion of machine parts expose to water and reduce machine efficiency.

Incorporation of Quorum Sensing inhibitors on the device surface is a possible strategy for declining $P$. aeruginosa biofouling of surgical implants. QS inhibition may be used to give defense against many pathogens that rely on QS to start biofilm development.

\section{Recombinant gene expression}

Possibly one of the exciting areas for research in quorum sensing is the synthesis of recombinant gene products with metabolic engineering. Quorum sensing has been used to control gene expression and cellular growth.

\section{Pathogen/pest management}

Pathogen and pest (i.e. some organism whose existence in a specific environment is undesirable) management include most of the present applications of quorum-sensing technology. Inhibition of quorum signalling is the evident and, in practice, most appreciated application of quorum-sensing knowledge. 


\section{New technologies in quorum sensing}

The discovery of antibiotics early in the past century marked the beginning of active control and prevention of infectious microbial diseases. However, extensive use of antibiotics has also unavoidably resulted in the emergence of 'superbugs' that resist conventional antibiotics. The finding that many pathogens rely on cell-to-cell communication mechanisms, known as quorum sensing, to synchronize microbial activities essential for infection and survival in the host suggests a promising disease control strategy, i.e. quenching microbial quorum sensing or in short, quorum quenching. Work over the past few years has demonstrated that quorum-quenching mechanisms are widely conserved in many prokaryotic and eukaryotic organisms. These naturally occurring quorum-quenching mechanisms appear to play important roles in microbe-microbe and pathogen-host interactions and have been used, or served as lead compounds, in developing and formulating a new generation of antimicrobials.

An advance study of bacterial quorum sensing process can facilitate development of novel technologies intended at interfering with bacterial communication and virulence.

The term "quorum sensing" explains the capability of a microorganism to recognize and response to diffusible signal molecules. Bacterial cells sense their inhabitant's density by a complicated cell-to-cell communication system also triggers expression of exact genes.

\section{Quorum sensing in seaweeds}

The quorum sensing is wider spread among bacterial population in Gram positive, Gramnegative bacterial communication. Followed by this have concluded that in Gram negative bacteria acyl-homoserine lactone is dependable for the cell to cell communication system.

In gram-positive bacteria peptide and derivative peptide based signalling molecules appear to be the main mode of communication. Throughout high cell density the marine bacteria can produce enzymes, surfactants, toxins, antibiotics by the chemical signal communication. Marine epibiotic bacteria are also identified to produce compounds active beside drug resistant host pathogen by the cross species induction process.

Quorum Sensing plays a main role in controlling a diversity of microbial cell activities like biofilm formation and virulence that considerably impact human health, agriculture, marine, commercial manufacture and transport systems. As mentioned in above applications of QS there are many areas that are fully touched by QS method. Therefore, significant research efforts are needed to understanding Quorum Sensing and the growth of strategies to disrupt and influence Quorum Sensing. More study and deep research is needed to uncover the details of QS in a diversity of microbial species, with Gram- positive bacteria and fungi. The task of QS in microbial populations, with Quorum Sensing crosstalk and signal specificity, is another significant area of research and study that will influence strategies to prevent biofilm formation and for biocontrol.

Quorum sensing seems to be a distinctive example of how the exploitation of bacteria cell-to-cell communication in biotechnology can be used to significantly drive the growth and development of medicine, diagnostics, therapies and gene control. For sure, it will influence every part of biology, with novel research and technologies in scientific world. 


\section{References}

Balagadde, F.K., Song, H., Ozaki, J., Collins, C.H., Barnet, M., Arnold, F.H., Quake, S.R. and You, L. 2008. A synthetic Escherichia coli predator-prey ecosystem. Molecular Systems Biology 4:187.

Basu, S., Gerchman, Y., Collins, C.H., Arnold, F.H. and Weiss, R. 2005. A synthetic multicellular system for programmed pattern formation. International Journal of Science 43(4): 1130-1134.

Brenner, K., Karig, D.K. and Arnold, F.H. 2007. Engineered bidirectional communication mediates a consensus in a microbial biofilm consortium. PNAS 104 (44): 17300-17304.

Fuqua, C., Parsek, M.R. and Greenberg, E.P. 2001. Regulation of Gene Expression by Cell-to-Cell Communication: ActylHomoserine Lactone Quorum Sensing. Annual Review of Genetics 35: 439468.

Joyce, E.A., Kawale A., Censini, S., Kim, C.C., Covacci, A. and Falkow, S. 2004. LuxS Is Required for Persistent Pneumococcal Carriage and Expression of Virulence and Biosynthesis Genes. Journal of Bacteriology 72(5): 29642975.

Kobayashi, H., Kaern, M., Araki, M., Chung, K., Gardner, T.S., Cantor, C.R. and Collins, J.J. 2014. Programmable cells: Interfacing natural and engineered gene networks. PNAS 101 (22): 8414-8419.

Kumari, A., Pasini, P. and Daunert. 2008. Detection of bacterial quorum sensing $N$ - acyl homoserine lactones in clinical samples. Analytical and Bioanalytical Chemistry 391(5): 1619-1627.

March, J.C. and Bentley, W.E. 2004. Quorum sensing and bacterial cross-talk in biotechnology. Current Opinion in Biotechnology 15: 495-502.
Muller, H., Westendorf, C., Leitner, E., Chernin, L., Riedel, K., Schmidt, S., Eberl, L. and Berg, G. 2009. Quorumsensing effects in the antagonistic rhizosphere bacterium Serratia plymuthica HRO-C48. FEMS Microbiology Ecology 67(3): 468-478.

Neddermann, P., Gargioli, C., Muraglia, E., Sambucini, S., Bonelli, F., Francesco, R.D. and Cortese, R. 2003. A novel, inducible, eukaryotic gene expression system based on the quorum- sensing transcription factor TraR. EMBO4: 159165.

Purnick, P.E.M. and Weiss, R. 2009. The second wave of synthetic biology: from modules to systems.Nature Reviews Molecular Cell Biology 10: 410-422.

Sanchez-Contreras, M., Bauer, W.D., Gao, M., Robinson and Downie, J.A. 2007. Quorum-sensing regulation in rhizobia and its role in symbiotic interactions with legumes. Philosophical Transactions of the Royal Society B 362.

Steindler, L. and Venturi, V. 2007. Detection of quorum-sensing $\mathrm{N}$-acyl homoserine lactone signal molecules by bacterial biosensors. FEMS Microbiology 266: 19.

Weber, W. and Fussenegger, M. 2009. Engineering of Synthetic Mammalian Gene Networks. Chemistry \& Biology 16(3): 287-297.

Weber, W., Malphettes, L., Jesus, M.D., Schoenmakers, R., Baba, M.D.EI., Spielmann, M., Keller, B., Weber, C.C., Wetering, P.V.D., Aubel, D., Wurm, F.M. and Fussenegger, M. 2005. Engineered Streptomyces quorum- sens ing components enable inducible siRNA- mediated translation control in mammalian cells and adjustable transcription control in mice. The Journal of Medicine 7(4): 518-525. 
William, C.N., DeShazer, D., Kim, S.H., Tettelin, H., Nelson, K.E., Feldblyum, T., Ulrich, R.L., Ronning, C.M., Brinkac, L.M., Daugherty, S.C., Davidsen, T.D., Deboy, R.T., Dimitrov, G., Dodson, R.J., Durkin, A.S., Gwinn, M.L., Haft, D.H., Khouri, H., Kolonay, J.F., Madupu, R., Mohammoud, Y., Nelson, W.C., Radune, D., Romero, C.M., Sarria, S., Selengut, J., Shamblin, C., Sullivan, S.A., White, O., Yu, Y.,
Zafar, N., Zhou, L. and Fraser, C.M. 2004. Structural flexibility in the Burkholderia mallei genome. PNAS 101(39): 14246-14251.

You, L., Cox, R.S., Weiss, R. and Arnold, F.H. 2004. Programmed population control by cell-cell communication and regulated killing. International Journal of Science 428: 868-871.

\section{How to cite this article:}

Malik Asif Aziz, Shazia Lone, Mushtaq Ah. Malik, Zaffar Mahdi Dar, Amjad Masood, Misbah Aijaz, Saima Shafi and Showkat Sidique. 2019. Quorum Sensing and Its Applications in Diverse Fields of Significance. Int.J.Curr.Microbiol.App.Sci. 8(07): 1272-1278. doi: https://doi.org/10.20546/ijcmas.2019.807.151 